\begin{tabular}{|c|c|}
\hline Title & Noninvasive and persistent transfollicular drug delivery system using a combination of liposomes and iontophoresis \\
\hline Author(s) & $\begin{array}{l}\text { Kai imoto, Kazuaki; Y amamoto, Masahiko; W atanabe, Misuzu; Kigasawa, Kaoru; Kanamura, Kiyoshi; Harashima, } \\
\text { Hidey oshi; Kogure, Kentaro }\end{array}$ \\
\hline Citation & $\begin{array}{l}\text { International Journal of Pharmaceutics, 403(1-2), 57-65 } \\
\text { https://doi.org/10.1016/.ijpharm.2010.10.021 }\end{array}$ \\
\hline Issue Date & 2011-01-17 \\
\hline Doc URL & http:/hdl.handle.net/2115/44921 \\
\hline Tyре & article (author version) \\
\hline Additional Information & There are other files related to this item in HUSCAP. Check the above URL. \\
\hline File Information & IJP403-1-2_57-65.pdf \\
\hline
\end{tabular}

Instructions for use 
International Journal of Pharmaceutics

Original Article

\section{Noninvasive and Persistent Transfollicular Drug Delivery System Using a Combination of Liposomes and Iontophoresis}

Kazuaki Kajimoto ${ }^{1}$, Masahiko Yamamoto $^{1}$, Misuzu Watanabe ${ }^{1}$, Kaoru Kigasawa ${ }^{1}$, Kiyoshi Kanamura $^{2}$, Hideyoshi Harashima ${ }^{1,3}$, Kentaro Kogure ${ }^{4, *}$

${ }^{1}$ Laboratory of Innovative Nanomedicine, Faculty of Pharmaceutical Sciences, Hokkaido University, Kita 12, Nishi 6, Kita-ku, Sapporo, Hokkaido 060-0812, Japan

${ }^{2}$ TTI ellebeau Inc., 4-8-8, Higashi-shinagawa, Shinagawa-ku, Tokyo, 140-0002, Japan

${ }^{3}$ Laboratory for Molecular Design of Pharmaceutics, Faculty of Pharmaceutical Sciences, Hokkaido University, Kita 12, Nishi 6, Kita-ku, Sapporo, Hokkaido 060-0812, Japan

${ }^{4}$ Department of Biophysical Chemistry, Kyoto Pharmaceutical University, Misasagi-Nakauchicho 5, Yamashina-ku, Kyoto 607-8414, Japan

Running Title: Iontophoresis for Delivery of Liposomes

*Correspondence:

Kentaro Kogure, Ph. D.

Department of Biophysical Chemistry, Kyoto Pharmaceutical University,

Misasagi-Nakauchicho 5, Yamashina-ku, Kyoto 607-8414 Japan

TEL: +81-75-595-4663

FAX: +81-75-595-4762

E-mail: kogure@mb.kyoto-phu.ac.jp 


\begin{abstract}
Iontophoresis is a promising technique for enhancing transdermal administration of charged drugs. However, conventional iontophoresis is not sufficient for effective delivery of large, hydrophilic, or electrically neutral molecules. In this study, we utilized charged liposomes as carriers, focused on a transfollicular route for delivery of the liposomes, and optimized iontophoretic conditions and lipid composition for this method in both in vitro and in vivo conditions. As a result, we identified the optimum condition (lipid composition: DOTAP/EPC/Chol=2:2:1, current supply: $0.45 \mathrm{~mA} / \mathrm{cm}^{2}$, duration: $1 \mathrm{hr}$ ) for effective iontophoretic delivery of aqueous solution, which can not be transferred into the skin without charged liposomes. We also examined the pharmacological effects of iontophoresis of liposomes encapsulating insulin (INS-lipo) using a rat model of type I diabetes. Interestingly, iontophoresis of INS-lipo onto a diabetes rat skin resulted in a gradual decrease in blood glucose levels, with levels reaching $20 \%$ of initial values at $18 \mathrm{hr}$ after administration. These lower blood glucose levels were maintained for up to $24 \mathrm{hr}$. Significant amount of insulin were also detected in plasma $18 \mathrm{hr}$ after iontophoresis of INS-lipo. We succeeded in developing a non-invasive and persistent transfollicular drug delivery system that used a combination of liposomes and iontophoresis.
\end{abstract}

\title{
Keywords
}

Liposomes, Iontophoresis, Transdermal delivery, Insulin, Diabetes 


\section{Abbreviations}

INS-lipo: insulin encapsulated in charged liposomes

DM: type I diabetes mellitus

NPH: neutral protamine Hagedorn

NBD: 4-nitrobenzo-2-oxa-1,3-diazolyl

DOPE: 1,2-dioleoyl phosphatidylethanolamine

DOTAP: 1,2-dioleoyl-3(trimethylammonium) propane

Chol: cholesterol

EPC: egg phosphatidylcholine

CHEMS: cholesteryl hemisuccinate

STZ: streptozotocin

SD: Sprague Dawley 


\section{Introduction}

Transdermal administration of drug molecules is considered to have numerous clinical benefits, such as avoiding the liver first-pass effect, improvement in patient compliance, and a reduction in adverse effects (Prausnitz and Langer, 2008). However, as the skin functions as a barrier in many cases, an innovative method for enhancing absorption of drug molecules across this barrier is required.

Iontophoresis utilizes an electric field to enhance the delivery of charged compounds across the skin and is recognized as a promising method for transdermal administration of drugs (Tyle, 1986; Varghese and Khar, 1996). Iontophoresis primarily provides an electrical driving force for transport of compounds across the stratum corneum, in addition to increasing skin permeability to drug molecules. As iontophoresis does not change the skin barrier directly, it is particularly applicable for transdermal administration of small, hydrophobic and charged molecules (Prausnitz and Langer, 2008).

In order to deliver drug molecules that are poorly delivered by iontophoresis, such as large, hydrophilic, or electrically neutral molecules, we investigated using charged liposomes as carriers for iontophoretic transdermal delivery. However, it was readily apparent these liposomes, generally having a diameter $>100 \mathrm{~nm}$, would not be able to penetrate the horny cell layer of the skin as they were larger than the intercellular spaces (Lasch et al., 1992). To overcome this problem, we considered the hair follicle may provide a suitable pathway for transdermal delivery of charged liposomes using iontophoresis.

There are some reports for follicular drug delivery using the nano- or microparticulate systems (several hundreds $\mathrm{nm}$ - several $\mu \mathrm{m}$ ) (Alvarez-Roman et al., 2004; Mordon et al., 2003; Rolland et al., 1993; Toll et al., 2004). While the follicular opening constitute only about $0.1 \%$ of the total skin area (Meidan et al., 2005), the hair follicle represents an invagination of the epidermis extending deep into the dermis and thus provides a greater actual area for potential absorption (Agarwal et al., 2000; Singh et al., 2000). 
Furthermore, there is no mature stratum corneum below the ostia of the sebaceuous glands, although the surfaces of the follicular openings are initially keratinised. In addition, there is an extensive capillary network associated with the upper dermal vasculature (Meidan et al., 2005). According to these features, the hair follicle might have great potential for drug delivery into the viable skin layers or the systemic circulation.

In this study, we combined liposome and iontophoresis technology to develop in vitro and in vivo non-invasive transdermal delivery systems. We also examined the in vivo effects of transfollicular administration of insulin encapsulated in liposomes in streptozotocin-induced diabetic model rats, by examining the prolonged action of insulin on blood glucose levels in these animals. 


\section{Materials and methods}

\subsection{Materials}

Sulforhodamine B was purchased from Molecular Probes (Carlsbad, CA, USA) and 4-nitrobenzo-2-oxa-1,3-diazolyl- (NBD-), Rhodamine- or non- labeled 1,2-dioleoyl phosphatidylethanolamine (DOPE), 1,2-dioleoyl-3(trimethylammonium) propane (DOTAP) and cholesterol (Chol) were obtained from Avanti Polar Lipids (Alabaster, AL, USA). Egg phosphatidylcholine (EPC) was purchased from NOF Corp. (Tokyo, Japan), and cholesteryl hemisuccinate (CHEMS) and insulin (porcine) from Sigma (St. Louis, MO, USA). The devices for iontophoresis $\left(\mathrm{TCT}^{\mathrm{TM}}\right.$ ) were manufactured by TTI Ellebeau Inc. (Tokyo, Japan).

\subsection{Preparation of liposomes}

DOTAP-based cationic liposomes and CHEMS-based anionic liposomes were prepared as described previously (Barichello et al., 2006). Briefly, 5 mol lipids: DOTAP/Chol (7:3 molar ratio), DOTAP/EPC $\quad(1: 1), \quad$ DOTAP/EPC/Chol $\quad(2: 2: 1), \quad$ DOTAP/DOPE $\quad(1: 1)$, DOTAP/EPC/DOPE (2:1:1), CHEMS/EPC (2:9), or CHEMS/DOPE (2:9) were mixed in chloroform and dried to a thin lipid film under vacuum using a rotary evaporator $(\mathrm{N}-1000$, EYELA, Tokyo, Japan). The dry lipid film was hydrated with $1 \mathrm{ml}$ of $10 \mathrm{mM}$ HEPES buffer $(\mathrm{pH}$ 7.4) to obtain a lipid suspension and the particles then downsized by sonication using a bath-type sonicator (AU-25C, Aiwa, Tokyo, Japan). In order to prepare liposomes with a defined size distribution, the sonicated particles were extruded through polycarbonate membranes (Whatman, pore size: 400 and then $100 \mathrm{~nm}$ ).

Liposomes encapsulating rhodamine or insulin were also prepared based on the same methodology. Namely, $2.5 \mathrm{mM}$ Sulfo rhodamine B or $2.4 \mathrm{mg} / \mathrm{ml}$ porcine insulin in $10 \mathrm{mM}$ HEPES buffer were added to the lipid film for hydration. For confocal studies, porcine insulin was fluorescently labeled using a NHS-Rhodamine Antibody Labeling Kit (Pierce) according to 
the manufacturer's protocol, while the lipid membrane of the liposomes was labeled using rhodamine- or NBD- labeled DOPE (5 mol\% of total lipids). In order to increase entrapment of insulin, the liposome suspension obtained was subjected to 6 freeze-thaw cycles. After sonication and extrusion, non-encapsulated rhodamine or insulin were separated from the liposome suspension by ultracentrifugation (Himac CS 150GX, HITACHI, Tokyo, Japan) at $50,000 \mathrm{x} \mathrm{g}$, at $4^{\circ} \mathrm{C}$ for $4 \mathrm{hr}$ or $30 \mathrm{~min}$, respectively. After preparation of liposomes, the recovered amount of insulin and phospholipids were determined by BCA method as described previously (Brown et al., 1989) and Phospholipids C test-Wako (Wako Pure Chemical, Osaka, Japan), respectively. The recovery ratio of insulin and lipid were calculated as the recovered insulin and lipid divided by the applied ones, multiplied by 100 .

The hydrodynamic diameter was measured by a quasi-elastic light scattering method and the zeta potential determined electrophoretically using an electrophoretic light scattering spectrophotometer (ELS-8000, Otsuka Electronics, Osaka, Japan). The physical characteristics of the liposomes used in the studies are shown in Tables I and II.

\subsection{Transfollicular delivery of liposomes using iontophoresis}

Shaved dorsal skins were excised from 10 week old male Sprague Dawley (SD) rats, and then held between the $\mathrm{TCT}^{\mathrm{TM}}$ devices (Kigasawa et al., 2010; Kigasawa et al., 2009) for in vitro iontophoresis (Fig. 1a). $1 \mathrm{ml}$ of $5 \mathrm{mM}$ suspensions of cationic or anionic liposomes $(5 \mu \mathrm{mol}$ lipids) was added to the anode or cathode sides of the drug chamber, respectively. A pair of $\mathrm{Ag}-\mathrm{AgCl}$ electrodes was then connected to a current power supplier (TCCR-3005, TTI ellebeau) and a current density of 0.3 or $0.45 \mathrm{~mA} / \mathrm{cm}^{2}$ applied for either 1 or $2 \mathrm{hr}$. The $\mathrm{TCT}^{\mathrm{TM}}$ devices were removed from the skin immediately after iontophoresis. Three hours after iontophoresis, a cross section of the skin (15 $\mu \mathrm{m}$ thickness) was observed by confocal laser scanning microscopy (LSM510, Carl Zeiss, Oberkochen, Germany). The delivery distances of rhodamine-labeled liposomes into the hair follicles were measured using the LSM Image 
Browser version 4.2.0 (Carl Zeiss).

For in vivo delivery of liposomes, the $\mathrm{TCT}^{\mathrm{TM}}$ devices were pasted onto the shaved dorsal skin of 10 week old male SD rats (Fig. 1b) and iontophoresis carried out under anesthesia at 0.45 $\mathrm{mA} / \mathrm{cm}^{2}$ for $1 \mathrm{hr}$. The $\mathrm{TCT}^{\mathrm{TM}}$ devices were removed from the skin immediately after iontophoresis. Three hours after iontophoresis, the rats were killed by $\mathrm{CO}_{2}$, followed by excision of the area of treated skin. The preparation of cross sections of skin and observation by confocal laser scanning microscopy were performed as described above.

\subsection{Fluorescent staining of micro vessels in rat skin}

The dorsal skins were excised from 10 week old male SD rats. The hairs were removed by shaving, followed by preparation of frozen sections ( $40 \mu \mathrm{m}$ thickness). The cross sections were washed in PBS and then stained with $10 \mu \mathrm{g} / \mathrm{ml}$ Fluorescein isothiocyanate (FITC)-labeled isolectin B4 [Griffonia (Bandeiraea) simplicifolia lectin I] (GSI-B4) (Vector Laboratories) for 1 $\mathrm{hr}$ and then $1 \mu \mathrm{g} / \mathrm{ml}$ Hoechst33342 (Nacalai Tesque) for $1 \mathrm{~min}$. After washing, the stained cross sections were observed using $15 \mu \mathrm{m}$-thick stacks of images obtained at $1 \mu \mathrm{m}$ intervals by confocal laser scanning microscopy (LSM510, Carl Zeiss).

\subsection{Experiment on streptozotocin (STZ)-induced diabetic model rats}

This experiment used 6-7 week old male SD rats. Induction of diabetes was performed as described previously (Kanikkannan et al., 1999). Briefly, a freshly prepared solution of streptozotocin (STZ) (Wako Pure Chemical, Osaka, Japan) (100 mg/kg) in $0.1 \mathrm{M}$ citrate buffer, pH 4.4 was injected intraperitoneally. Blood glucose levels were determined $48 \mathrm{hr}$ after STZ administration using a blood glucose monitoring system (Glucose Pilot, Technicon International, Tokyo, Japan). If the blood glucose level was $>300 \mathrm{mg} / \mathrm{dl}$, the animal was considered to have had successful induction of diabetes and was used in the study. 


\subsection{In vivo delivery of insulin encapsulated in liposomes (INS-lipo)}

The STZ-induced diabetic SD rats were fasted overnight until the start of the experiment. A catheter was implanted in the jugular vein of the fasted rats under anesthesia, and then the iontophoretic device $\mathrm{TCT}^{\mathrm{TM}}$ filled with a suspension of INS-lipo was pasted onto the shaved dorsal skin and iontophoresis performed as described above (i.e. $0.45 \mathrm{~mA} / \mathrm{cm}^{2}, 1 \mathrm{hr}$ ). The amount of insulin and total lipids filled in $\mathrm{TCT}^{\mathrm{TM}}$ devices were approximately $0.66 \mathrm{mg} / \mathrm{kg}(18.3$ $\mathrm{IU} / \mathrm{kg}$ ) and $4.7 \mu \mathrm{mol} / \mathrm{kg}$, respectively. Blood samples were drawn from the catheter using the Auto Blood Sampling System (DR-II, EICOM Co., Ltd., Kyoto, Japan) at 0, 1, 3, 6, 9, 12, and $18 \mathrm{hr}$ after iontophoresis had been started. During the period of blood sampling, the fasted rats were allowed access to water ad libitum. As a positive control experiment, a $180 \mu \mathrm{g} / \mathrm{kg}(5$ $\mathrm{IU} / \mathrm{kg}$ ) dose of insulin solution was injected intraperitoneally in the diabetic rats, and blood samples collected via an implanted catheter in the jugular vein every $1 \mathrm{hr}$ for either $6 \mathrm{hr}$ or $18 \mathrm{hr}$ after injection. Blood glucose levels were determined in all the samples as described above.

\subsection{Plasma insulin concentration}

The blood samples were centrifuged for $15 \mathrm{~min}$ at $700 \mathrm{x}$ g and the separated plasma stored at $-20^{\circ} \mathrm{C}$ until assayed. Plasma insulin was assayed using a porcine insulin ELISA kit (Mercodia, Uppsala, Sweden) according to the manufacturer's protocol. The detection limit of the ELISA assay kit used in the study was $0.01 \mathrm{ng} / \mathrm{ml}$.

\subsection{Statistical Analysis}

Statistical analysis of the differences between treatments was carried out using unpaired Student's t-tests. P-values $<0.05$ were considered significant. 


\section{Results}

\subsection{Optimization of conditions for in vitro transfollicular delivery of liposomes via iontophoresis}

At first, we determined whether it was possible to deliver liposomes into hair follicles using iontophoresis. Confocal laser scanning microscopy was used to observe cross sections of the skin after in vitro iontophoresis of liposomes in which the lipid membrane was labeled with rhodamine. Cationic liposomes, composed of DOTAP/Chol (7:3), and several iontophoretic conditions of current density and duration were attempted to use for delivery of the liposomes into the hair follicles. As shown in Fig. 2, the lipid marker of liposomes achieved significantly greater delivery distances into the follicles at conditions of $0.45 \mathrm{~mA} / \mathrm{cm}^{2}$ for $1 \mathrm{hr}$ compared to the other experimental conditions used. This finding demonstrated charged liposomes can be delivered into hair follicles using iontophoresis.

Next, we examined the influence of lipid composition of the liposomes on delivery distance into the hair follicles. Both the lipid marker of cationic and anionic charged liposomes penetrated the hair follicles during iontophoresis, with the exception of cationic liposomes containing the fusogenic lipid DOPE (Fig. 3a). However, the lipid marker of liposomes containing DOPE were shown to penetrate the entire skin surface. The lipid marker of cationic liposomes reached a deeper region of the hair follicles than that of anionic liposomes (Fig. 3b). In particular, the delivery distance of the lipid marker of cationic liposomes consisting of DOTAP, EPC, and Chol (2:2:1) into hair follicles tended to be higher than that of DOTAP/Chol (7:3) and was significantly higher than the other liposomes examined.

In all the above experiments we used liposomes with mean diameters of approximately 100 $150 \mathrm{~nm}$. To evaluate whether liposomal size affected the delivery distance into hair follicles, we prepared liposomes composed of DOTAP/EPC/Chol (2:2:1) that had a diameter range of $100-400 \mathrm{~nm}$ and subjected them to iontophoretic delivery. This showed that variation in liposome sizes did not significantly affect delivery distance into the hair follicles (data not 
shown).

From these results, it was concluded the optimal iontophoretic conditions and lipid compositions were $0.45 \mathrm{~mA} / \mathrm{cm}^{2}$ for $1 \mathrm{hr}$ and DOTAP/EPC/Chol $(2: 2: 1)$. The following experiments were performed using these optimal conditions.

\subsection{Liposomes as a drug carrier for transfollicular delivery via iontophoresis}

To confirm the function of liposomes as a drug carrier for transfollicular delivery via iontophoresis, we prepared double labeled liposomes, consisting of an aqueous phase labeled with rhodamine (red) and a lipid membrane labeled with NBD (green). We then examined whether the lipid membrane and the aqueous phase were both delivered into hair follicles after in vitro (Fig. 4a) and in vivo (Fig. 4b) iontophoresis.

As shown in Fig. 4a, in the case of free rhodamine solution (Rho-soln), rhodamine dye was observed only on the skin surface and was not delivered into the hair follicles by iontophoresis. On the other hand, rhodamine was clearly detected in hair follicles when the fluorescent dye was encapsulated in cationic liposomes (Rho-lipo). In addition, NBD-lipid was also observed in the hair follicles. These results suggest strongly that liposomes can function as a carrier for transfollicular delivery via iontophoresis.

We also observed NBD-labeled liposomes encapsulating rhodamine after in vivo iontophoresis. Delivery of rhodamine and NBD-lipid into the hair follicles was also demonstrated after in vivo iontophoresis (Fig. 4b). Interestingly, after in vivo iontophoresis, rhodamine and NBD fluorescence was observed around the hair follicles and distributed widely in the skin, whereas following in vitro iontophoresis this was localized in the hair follicles.

\subsection{In vivo iontophoretic transfollicular delivery of liposomes encapsulating insulin}

As it appeared it was possible to use liposomes and iontophoresis to deliver drug molecules into hair follicles, we next attempted to deliver functional large molecules using this system. 
For these investigations we selected insulin as a model, and observed NBD-labeled liposomes encapsulating rhodamine-labeled insulin after in vivo iontophoresis. The recovery ratio of insulin and lipid after preparation of liposomes were approximately $65 \%$ and $22 \%$, respectively.

The solution of rhodamine-labeled insulin (INS-soln) or liposomes encapsulating insulin (INS-lipo) were subjected to iontophoretic administration from the anode side. As shown in Fig. 5a, we achieved insufficient delivery of INS-soln alone into the hair follicles. However, in the case of INS-lipo, the fluorescence of rhodamine-labeled insulin was clearly detected in the epidermal region including the stratum corneum, with small amounts being observed in or surrounding the hair follicles. Interestingly, $18 \mathrm{hr}$ after iontophoresis, the fluorescence of rhodamine-labeled insulin was still observed in a part of the epidermal region and hair follicles. These results suggest that our system combining liposomes with iontophoresis is applicable for delivering macromolecules through hair follicles.

We also examined the distribution of micro vessels in the skin to determine the distance between hair follicles and blood vessels. As shown in Fig. 5b and Supplementary Fig. 1, we confirmed that micro vessels were distributed densely in the vicinity of hair follicles and the basal cell layer in the dermis. These results suggest that drug molecules possibly enter the blood circulation via micro vessels in these regions, even if they have not been delivered to deeper regions of the skin.

\subsection{Effect of in vivo iontophoretic transfollicular delivery of INS-lipo on blood glucose levels in type I diabetic model rats}

We next examined the effect of transfollicular delivery of INS-lipo using iontophoresis on blood glucose levels in streptozotocin (STZ)-induced type I diabetic rats. The mean fasting blood glucose level of the diabetic rats was $403 \pm 56 \mathrm{mg} / \mathrm{dl}$, compared to $123 \pm 6 \mathrm{mg} / \mathrm{dl}$ in non-treated rats. As a positive control experiment, an i.p. injection of insulin administered to the diabetic rats caused an immediate decrease in blood glucose levels (Fig. 6a). However, 
blood glucose levels began to increase $4 \mathrm{hr}$ after the insulin injection and returned to basal levels $18 \mathrm{hr}$ after injection. In contrast, with iontophoresis of INS-lipo, blood glucose levels decreased slowly after iontophoretic administration, reducing to approximately $20 \%$ of basal levels $18 \mathrm{hr}$ after iontophoresis (Fig. 6a). These lower blood glucose levels were maintained for up to $24 \mathrm{hr}$ after administration (data not shown). In addition, the amount of insulin remained in $\mathrm{TCT}^{\mathrm{TM}}$ devices immediately after iontophoresis was approximately $80 \%$ of initial amount. Moreover, iontophoresis of empty liposomes (Emp-lipo) did not affect blood glucose levels during this period (Fig. 6a).

\subsection{Measurement of plasma insulin levels after iontophoresis of INS-lipo}

We then used an ELISA assay to measure plasma insulin concentrations in diabetic rats after iontophoresis of INS-lipo (Fig. 6b). As a positive control experiment, an i.p. injection of insulin administered to the diabetic rats caused an immediate increase in plasma insulin concentrations up to $11.6 \pm 1.56 \mathrm{ng} / \mathrm{ml} 1 \mathrm{hr}$ after injection. However, $18 \mathrm{hr}$ after injection, plasma insulin levels decreased significantly and returned to approximately basal levels $(0.22 \pm$ $0.31 \mathrm{ng} / \mathrm{ml}$ ). One hour after iontophoresis, the plasma insulin concentrations of the rats treated with INS-lipo or empty liposomes were $0.28 \pm 0.21$ and $0.05 \pm 0.03 \mathrm{ng} / \mathrm{ml}$, respectively. At 18 $\mathrm{hr}$ after iontophoresis, mean plasma insulin concentrations in the rats treated with INS-lipo had increased to $1.41 \pm 0.84 \mathrm{ng} / \mathrm{ml}$ and were significantly higher than the concentrations measured with either empty liposomes $(0.17 \pm 0.07 \mathrm{ng} / \mathrm{ml})$ or i.p. insulin injection. These results are in accordance with the blood glucose levels measured, indicating that transdermal delivery of liposomes encapsulating insulin, facilitated by iontophoresis, successfully supplies insulin to the circulatory system. 


\section{Discussion}

In vitro anodal iontophoresis of cationic liposomes (DOTAP/Chol $=7: 3$ ) achieved maximum delivery distance into hair follicles at conditions of $0.45 \mathrm{~mA} / \mathrm{cm}^{2}$ for $1 \mathrm{hr}$ (Fig. 2). In the condition of $0.45 \mathrm{~mA} / \mathrm{cm}^{2}$ for $2 \mathrm{hr}$, delivery distance into hair follicles of liposomes was shorter than that of $0.45 \mathrm{~mA} / \mathrm{cm}^{2}$ for $1 \mathrm{hr}$ (Fig. 2). Probably, some parts of the liposomes delivered into hair follicles during first $1 \mathrm{hr}$ treatment were excluded from the hair follicles by impairments, such as inflammation, induced by longer duration of iontophoresis. The application of higher current and longer duration caused impairments in skin structure that made it difficult to prepare thin sections for confocal microscopy (data not shown). These results are in accordance with a previous report that burning and skin irritation are induced at a current density of more than $0.5 \mathrm{~mA} / \mathrm{cm}^{2}$ (Ledger, 1992).

As shown in Table I, the size of liposomes examined here and their homogeneity were very similar. The electrical charge and flexibility of liposomes depends on their lipid composition. In order to optimize the lipid composition of liposomes for transfollicular delivery by iontophoresis, we investigated several DOTAP-based cationic or CHEMS-based anionic liposomes (Fig. 3). The results showed cationic liposomes containing Chol $(\mathrm{DOTAP} / \mathrm{EPC} / \mathrm{Chol}=2: 2: 1)$ were delivered into hair follicles to a significantly greater depth than the other liposome preparations. It is difficult to distinguish the liposomes penetrating through the hair follicles from those accumulating in the follicles. However, it was obvious that cationic-liposomes containing DOPE were not only transfer into the hair follicles but penetrate through the skin surface. It has been reported that Chol stabilizes liposomal membranes (Damen et al., 1981; Kirby et al., 1980), whereas DOPE, a fusogenic lipid, destabilizes the lipid bilayers by promoting transition from a lamellar to a hexagonal II phase (Guo and Szoka, 2001). As shown in Table I, although the physical characteristics (size, $\zeta$-potential and polydispersity) of cationic liposomes consisted of DOTAP/EPC $=1: 1$ and $\mathrm{DOTAP} / \mathrm{EPC} / \mathrm{DOPE}=2: 1: 1$ were very similar, the distribution of lipid marker in the skin after 
iontophoresis was entirely different (Fig. 3a). This suggests that appropriate stability of liposomes is an important determinant of transfollicular delivery by iontophoresis. Furthermore, we found cationic liposomes achieved greater delivery distances than anionic liposomes. This difference was probably a consequence of the negative charge on the surface of the skin (Burnette and Ongpipattanakul, 1987) and electro-osmotic flow from the anode to the cathode during iontophoresis (Pikal, 1992; Sieg et al., 2004). In order to elucidate the reason for why anionic liposomes examined in this study was not delivered into the deeper region of the hair follicles compared with cationic liposomes, further investigations about the influence of lipid composition of liposomes or electric potential are required.

Moreover, some of cationic- or anionic-liposomes (lipid marker) spread over the stratum corneum 3 hrs after iontophoresis (Figs. 2a and 3a). These results are well consistent with the previous report. Lasch $\mathrm{J}$ et al. have described that both fluorescent-labeled lipid and aqueous markers of liposomes spread over the horny layer within $30 \mathrm{~min}$ after pipetting them onto excised human skin, but do not penetrate deeper than the horny cell layer even after $24 \mathrm{hrs}$ (Lasch et al., 1992). From these findings, it was suggested that liposomes can not defuse or penetrate into dermis through the stratum corneum under the passive condition.

As shown in Table II, although the $\zeta$-potential of liposomes encapsulating rhodamine or insulin was not so different from empty liposomes shown in table I, the average size of those was larger than empty liposomes. Our investigations on the effect of liposomal size revealed there was no significant difference in transfollicular delivery between small $(100-400 \mathrm{~nm})$ and large (>400 nm) liposomes (data not shown). In vitro and in vivo assessments using double fluorescent labeling liposomes also revealed that both NBD-lipid and rhodamine encapsulated in liposomes were delivered into hair follicles after iontophoresis (Fig. 4). However, iontophoresis of free rhodamine solution showed no delivery of the fluorescent marker into the hair follicles, indicating that charged liposomes function as a carrier of rhodamine for transfollicular delivery via iontophoresis. Furthermore, NBD-lipid and rhodamine were both 
distributed widely in the skin via hair follicles only following in vivo administration. As rhodamine dye alone was not transferred by iontophoresis, it is possible that part of the liposomes decayed after delivery to the hair follicles, resulting in release of rhodamine that penetrated into the skin. Although the setting of iontophoresis such as a current density and duration was same between in vitro and in vivo experiments, the placements of electrodes are different. Therefore, the difference might affect the mode of current flow in the rat skin. Particularly, in case of the in vitro iontophoresis, the skin was hold between the anode and cathode, hence, the current flow might be greater than in vivo iontophoresis. There are some reports describing that liposomes have been used to enhance transfollicular and transdermal delivery of the low-/high-molecular weight and hydrophilic/lipophilic compounds (Fleisher et al., 1995; Li and Hoffman, 1996; Niemiec et al., 1997; Niemiec et al., 1995; Seiberg et al., 1997). However, we have confirmed that in vivo permeation of liposomes from the hair follicles into dermis/epidermis was not appeared under passive condition (Supplementary Fig. 2). Therefore, the combination of liposomes and iontophoresis might be further enhancing the permeation through the hair follicles. The molecular basis of such a synergistic effect remains unclear and requires further investigation.

In order to evaluate the application of transfollicular delivery of liposomes encapsulating functional macromolecules, we prepared liposomes encapsulating rhodamine-labeled insulin, in which the lipid membrane was labeled fluorescently by NBD-DOPE prior to in vivo iontophoresis. Similar to rhodamine dye, insulin alone was not delivered sufficiently into the hair follicles by anodal iontophoresis due to its negative charge under these conditions (Fig. 5a). However, insulin encapsulated in cationic liposomes was delivered successfully into the epidermal region including the stratum corneum and marginally in or around the hair follicles. Surprisingly, $18 \mathrm{hr}$ after iontophoresis, the fluorescence of rhodamine-labeled insulin was still recognized in a part of the epidermal region and hair follicles. It has been estimated that the half-life of insulin in the body is about 5 min (Duckworth et al., 1998), and therefore it is 
possible that the lipid membrane protects insulin from degradation by proteases, or alternatively, that the stability of insulin in the stratum corneum or hair follicles is relatively higher than that in other parts of the body such as the circulation. Furthermore, we demonstrated micro vessels were distributed densely in the vicinity of hair follicles and basal cell layer in the dermis (Fig. 5b). These results suggest that insulin possibly enters the blood circulation via micro vessels from the stratum corneum and hair follicles, even if it has not been delivered to deeper regions of the skin. It is also possible that administration of INS-lipo by iontophoresis causes a persistent effect.

In order to examine this possibility, STZ-induced diabetic rats were administered regular porcine insulin encapsulated in liposomes by iontophoresis. Control i.p. injections of insulin solution showed the lowest blood glucose levels were attained $4 \mathrm{hr}$ after administration, followed by a gradual increase (Fig. 6a). Similar results have been described previously (Ito et al., 1999; Lee et al., 2003). Interestingly, blood glucose levels decreased gradually after iontophoretic administration of INS-lipo, with levels reducing to approximately $20 \%$ of basal levels $18 \mathrm{hr}$ after iontophoresis. These low levels were maintained for at least $24 \mathrm{~h}$ after administration (data not shown). The maximum effect on blood glucose levels achieved with INS-lipo was the same as those with i.p. injections of insulin solution. This suggests that iontophoresis of charged liposomes is an efficient, non-invasive method for delivering insulin for diabetic therapy. In this experiment, blood glucose levels were measured under fasting conditions in order to minimize the influence of feeding on blood glucose, and therefore, the measurements were carried out up to $24 \mathrm{~h}$ after iontophoresis. To obtain further information on the long-term action of INS-lipo in diabetic animals we also need to carry out the experiments under normal feeding conditions.

The plasma insulin level of the STZ-induced diabetic rats was not detectable prior to the liposome experiments. However, $1 \mathrm{hr}$ after i.p. injection of insulin solution, plasma levels rose significantly and then decreased to approximately basal levels $18 \mathrm{hr}$ after injection (Fig. 6b). 
On the other hand, insulin levels increased to about $1.4 \mathrm{ng} / \mathrm{ml} 18 \mathrm{hr}$ after iontophoresis of INS-lipo, with this level being significantly higher than that achieved by i.p. injection. Interestingly, we found the highest insulin concentrations $18 \mathrm{hr}$ after iontophoresis, with these levels being significantly higher than levels measured immediately after the procedure (Fig. 6b). These results suggest that insulin delivered by liposomes via iontophoresis is released into the skin around hair follicles, and is then transferred gradually to the blood via the capillaries. In general, the plasma insulin concentration correlates with the blood glucose level. In case of subcutaneous injection of insulin solution, the plasma concentration increased transiently but rapidly recovered to the normal level, since the half life of insulin in blood is about $5 \mathrm{~min}$ (Duckworth et al., 1998). On the other hand, in case of transfollicular delivery via the combination of liposomes and iontophoresis, the reservoir effect in the skin and the sustained release from liposomes might contribute to maintain the not so high but active plasma concentration of insulin. The amount of insulin released from $\mathrm{TCT}^{\mathrm{TM}}$ devices immediately after iontophoresis was estimated at approximately $20 \%$ of initial amount. However, it was difficult to accurately estimate how many amount of insulin were administered into the body, hence, further investigations are required for the determination of bioavailability. Previous studies have reported fasting plasma insulin levels in normal rats range between $0.5-3.0 \mathrm{ng} / \mathrm{ml}$ (Chang et al., 1998; Tung et al., 2005). This basal level of insulin was maintained in our experimental rats for long periods of time following iontophoresis of INS-lipo. The persistent action shown in this study is useful for substitution of basic insulin secretion in the treatment of type 1 diabetes. However, only the persistent action is not necessarily sufficient for the strict control of blood glucose by insulin substitution. The administration system obtaining the instantaneous action remains further investigation. Moreover, the combination of the conventional insulin formulation for the instantaneous action and our persistent system might be available.

Previously, Han et al. also reported that transfollicular delivery of drug molecules was 
enhanced by using a combination of liposomes and iontophoresis (Han et al., 2005; Han et al., 2004). In those reports, the intrafollicular delivery of small fluorescent drug molecules was investigated. However, it was unclear whether liposomes were delivered into hair follicles with drugs. In addition, there is no report on successful systemic delivery of functional macromolecules such as insulin using a combination of liposomes and iontophoresis.

In the present study, this system was examined using rat skin, of which number of hair follicles are known to be higher than human skin. Therefore, in the future, improvement of the delivery efficiency of this system would be required for clinical application due to considering number of hair follicles in human skin.

In conclusion, our results demonstrate that transfollicular delivery of liposomes encapsulating drug molecules using iontophoresis may be applicable to various medicines and is not restricted to small molecules. This is the first report demonstrating the ability of liposomes to act as carriers for intradermal delivery of drugs. We also report successful transfollicular delivery of functional macromolecules using a combination of liposomes and iontophoresis. 


\section{Acknowledgments}

This work was supported in part by Grants-in-Aid for Scientific Research from the Ministry of Education, Culture, Sports, Science and Technology (MEXT) of Japan. 


\section{References}

Agarwal R., Katare O.P., Vyas S.P., 2000. The pilosebaceous unit: A pivotal route for topical drug delivery. Methods Find. Exp. Clin. Pharmacol., 22, 129-133.

Alvarez-Roman R., Naik A., Kalia Y.N., Guy R.H., Fessi H., 2004. Skin penetration and distribution of polymeric nanoparticles. J. Control. Release, 99, 53-62.

Barichello J.M., Handa H., Kisyuku M., Shibata T., Ishida T., Kiwada H., 2006. Inducing effect of liposomalization on the transdermal delivery of hydrocortisone: Creation of a drug supersaturated state. J. Control. Release, 115, 94-102.

Brown R.E., Jarvis K.L., Hyland K.J., 1989. Protein measurement using bicinchoninic acid: Elimination of interfering substances. Anal. Biochem., 180, 136-139.

Burnette R.R., Ongpipattanakul B., 1987. Characterization of the permselective properties of excised human-skin during iontophoresis. J. Pharm. Sci., 76, 765-773.

Chang C.J., Wu J.S., Lu F.H., Liu I.M., Chi T.C., Cheng J.T., 1998. Sympathetic hyperactivity in wistar rats with insulin-resistance. J. Auton. Nerv. Syst., 74, 116-119.

Damen J., Regts J., Scherphof G., 1981. Transfer and exchange of phospholipid between small unilamellar liposomes and rat plasma high-density lipoproteins - dependence on cholesterol content and phospholipid-composition. Biochim. Biophys. Acta, 665, 538-545.

Duckworth W.C., Bennett R.G., Hamel F.G., 1998. Insulin degradation: Progress and potential. Endocr. Rev., 19, 608-624.

Fleisher D., Niemiec S.M., Oh C.K., Hu Z., Ramachandran C., Weiner N., 1995. Topical delivery of growth hormone releasing peptide using liposomal systems: An in vitro study using hairless mouse skin. Life Sci., 57, 1293-1297.

Guo X., Szoka F.C., 2001. Steric stabilization of fusogenic liposomes by a low-ph sensitive peg-diortho ester-lipid conjugate. Bioconjug. Chem., 12, 291-300.

Han I., Jun M.S., Kim S.K., Kim M., Kim J.C., 2005. Expression pattern and intensity of protoporphyrin ix induced by liposomal 5-aminolevulinic acid in rat pilosebaceous unit 
throughout hair cycle. Arch. Dermatol. Res., 297, 210-217.

Han I., Kim M., Kim J., 2004. Enhanced transfollicular delivery of adriamycin with a liposome and iontophoresis. Exp. Dermatol., 13, 86-92.

Ito M., Kondo Y., Nakatani A., Naruse A., 1999. New model of progressive non-insulin-dependent diabetes mellitus in mice induced by streptozotocin. Biol. Pharm. Bull., 22, 988-989.

Kanikkannan N., Singh J., Ramarao P., 1999. Transdermal iontophoretic delivery of bovine insulin and monomeric human insulin analogue. J. Control. Release, 59, 99-105.

Kigasawa K., Kajimoto K., Hama S., Saito A., Kanamura K., Kogure K., 2010. Noninvasive delivery of sirna into the epidermis by iontophoresis using an atopic dermatitis-like model rat. Int. J. Pharm., 383, 157-160.

Kigasawa K., Kajimoto K., Watanabe M., Kanamura K., Saito A., Kogure K., 2009. In vivo transdermal delivery of diclofenac by ion-exchange iontophoresis with geraniol. Biol. Pharm. Bull., 32, 684-687.

Kirby C., Clarke J., Gregoriadis G., 1980. Effect of the cholesterol content of small unilamellar liposomes on their stability invivo and invitro. Biochem. J., 186, 591-598.

Lasch J., Laub R., Wohlrab W., 1992. How deep do intact liposomes penetrate into human skin. J. Control. Release, 18, 55-58.

Ledger P.W., 1992. Skin biological issues in electrically enhanced transdermal delivery. Adv. Drug Delivery. Rev., 9, 289-307.

Lee J.J., Yi H.Y., Yang J.W., Shin J.S., Kwon J.H., Kim C.W., 2003. Characterization of streptozotocin-induced diabetic rats and pharmacodynamics of insulin formulations. Biosci. Biotechnol. Biochem., 67, 2396-2401.

Li L.N., Hoffman R.M., 1996. Topical liposome-targeted selective delivery of molecules to hair follicles in mice. J. Invest. Dermatol., 106, 663-663.

Meidan V.M., Bonner M.C., Michniak B.B., 2005. Transfollicular drug delivery--is it a reality? 
Int. J. Pharm., 306, 1-14.

Mordon S., Sumian C., Devoisselle J.M., 2003. Site-specific methylene blue delivery to pilosebaceous structures using highly porous nylon microspheres: An experimental evaluation. Lasers Surg. Med., 33, 119-125.

Niemiec S.M., Latta J.M., Ramachandran C., Weiner N.D., Roessler B.J., 1997. Perifollicular transgenic expression of human interleukin-1 receptor antagonist protein following topical application of novel liposome-plasmid DNA formulations in vivo. J. Pharm. Sci., 86, 701-708.

Niemiec S.M., Ramachandran C., Weiner N., 1995. Influence of nonionic liposomal composition on topical delivery of peptide drugs into pilosebaceous units - an in-vivo study using the hamster ear model. Pharm. Res., 12, 1184-1188.

Pikal M.J., 1992. The role of electroosmotic flow in transdermal iontophoresis. Adv. Drug Delivery. Rev., 9, 201-237.

Prausnitz M.R., Langer R., 2008. Transdermal drug delivery. Nat. Biotechnol., 26, 1261-1268.

Rolland A., Wagner N., Chatelus A., Shroot B., Schaefer H., 1993. Site-specific drug delivery to pilosebaceous structures using polymeric microspheres. Pharm. Res., 10, 1738-1744.

Seiberg M., Wisniewski S., Cauwenbergh G., Shapiro S.S., 1997. Trypsin-induced follicular papilla apoptosis results in delayed hair growth and pigmentation. Dev. Dyn., 208, 553-564.

Sieg A., Guy R.H., Delgado-Charro M.B., 2004. Electroosmosis in transdermal iontophoresis: Implications for noninvasive and calibration-free glucose monitoring. Biophys. J., 87, $3344-3350$.

Singh P., Sihorkar V., Jaitely V., Kanaujia P., Vyas S., 2000. Pilosebaceous unit: Anatomical considerations and drug delivery opportunities. Indian J. Pharmacol., 32, 269-281.

Toll R., Jacobi U., Richter H., Lademann J., Schaefer H., Blume-Peytavi U., 2004. Penetration profile of microspheres in follicular targeting of terminal hair follicles. J. Invest. Dermatol., 123, 168-176.

Tung Y.C., Hewson A.K., Carter R.N., Dickson S.L., 2005. Central responsiveness to a ghrelin 
mimetic (ghrp-6) is rapidly altered by acute changes in nutritional status in rats. J. Neuroendocrinol., 17, 387-393.

Tyle P., 1986. Iontophoretic devices for drug delivery. Pharm. Res., 3, 318-326.

Varghese E., Khar R.K., 1996. Enhanced skin permeation of diclofenac by iontophoresis: In vitro and in vivo studies. J. Control. Release, 38, 21-27. 


\section{Tables}

Table I. Physical properties of the liposomes used to establish optimal in vitro conditions.

\begin{tabular}{llll}
\hline \hline Lipid composition (molar ratio) & Size $(\mathrm{nm})$ & $\xi$-potential $(\mathrm{mV})$ & Polydispersity \\
\hline DOTAP/Chol (7:3) & $112.9 \pm 2.0$ & $29.3 \pm 10.5$ & $0.073 \pm 0.016$ \\
DOTAP/EPC (1:1) & $107.5 \pm 1.9$ & $37.7 \pm 5.2$ & $0.092 \pm 0.009$ \\
DOTAP/EPC/Chol (2:2:1) & $116.6 \pm 3.2$ & $40.5 \pm 8.1$ & $0.079 \pm 0.009$ \\
DOTAP/DOPE (1:1) & $113.4 \pm 3.6$ & $51.9 \pm 2.2$ & $0.085 \pm 0.032$ \\
DOTAP/EPC/DOPE (2:1:1) & $111.2 \pm 3.3$ & $34.5 \pm 13.9$ & $0.100 \pm 0.026$ \\
CHEMS/EPC (2:9) & $108.1 \pm 4.5$ & $-23.6 \pm 11.8$ & $0.081 \pm 0.012$ \\
CHEMS/DOPE (2:9) & $152.9 \pm 1.7$ & $-61.1 \pm 10.3$ & $0.168 \pm 0.009$ \\
\hline \hline
\end{tabular}

Data represent the mean \pm SD of three independent experiments. 
Table II. Physical properties of liposomes encapsulating rhodamine or insulin.

\begin{tabular}{llll}
\hline \hline & Size $(\mathrm{nm})$ & $\zeta$-potential $(\mathrm{mV})$ & Polydispersity \\
\hline Rho-lipo $^{\mathrm{a}}$ & $288.5 \pm 55.9$ & $61.2 \pm 23.9$ & $0.248 \pm 0.016$ \\
INS-lipo $^{\mathrm{b}}$ & $243.4 \pm 17.6$ & $63.1 \pm 13.1$ & $0.120 \pm 0.030$ \\
\hline \hline
\end{tabular}

Data represent the mean \pm SD of 5 or 7 independent experiments. The lipid composition of both liposomes was DOTAP/EPC/Chol=2:2:1. ${ }^{a}$ Rho-lipo: liposomes encapsulating sulfo rhodamine B; ${ }^{\mathrm{b}}$ INS-lipo: liposomes encapsulating porcine insulin. 


\section{Figure legends}

Figure 1. Schematic images of the iontophoretic system $\left(\mathrm{TCT}^{\mathrm{TM}}\right)$ for transfollicular delivery of liposomes.

This system transfers charged liposomes from the drug chamber into hair follicles. The electrode and drug chamber were partitioned by an ion-exchange membrane to minimize undesirable interactions between the electrode and the drugs. In order to achieve effective delivery of liposomes to the skin, an ion-exchange membrane was not inserted between the skin and drug chamber. (a) For in vitro iontophoresis, the shaved and excised dorsal skin was held between the $\mathrm{TCT}^{\mathrm{TM}}$ devices. The suspensions of cationic or anionic liposomes were added to the anodal or cathodal drug chambers, respectively. (b) For in vivo delivery of liposomes, the $\mathrm{TCT}^{\mathrm{TM}}$ device was pasted onto the shaved dorsal skin.

Figure 2. Optimization of transfollicular delivery of DOTAP/Chol liposomes in vitro.

(a) Cross sections of skin were observed by confocal laser scanning microscopy $3 \mathrm{hr}$ after iontophoresis of rhodamine-labeled liposomes (red) composed of DOTAP/Chol $=7: 3$. Scale bar $=100 \mu \mathrm{m}$. (b) The delivery distances of rhodamine-labeled liposomes into the hair follicles were measured in microscopic images of randomly selected cross sections $(n=5-7)$ using LSM Image Browser software. Data are expressed as mean \pm SD. ND means not detected. $\quad * \mathrm{p}<0.05, * * \mathrm{p}<0.01$

Figure 3. Optimization of lipid composition for in vitro transfollicular delivery.

(a) Cross sections of skin were observed by confocal laser scanning microscopy $3 \mathrm{hr}$ after iontophoresis of rhodamine-labeled liposomes (red). Scale bar $=100 \mu \mathrm{m} . \quad$ (b) The delivery distances of rhodamine-labeled liposomes into the hair follicles were measured in microscopic images of randomly selected cross sections $(n=5-7)$ using LSM Image Browser software. Data are expressed as mean $\pm \mathrm{SD} . \quad$ ND means not detected. $\quad * \mathrm{p}<0.05, * * \mathrm{p}<0.01$ 
Figure 4. Transfollicular delivery of double labeled liposomes via iontophoresis.

In order to evaluate whether the lipid membrane and aqueous phase encapsulated in charged liposomes were both transferred by iontophoresis, we observed liposomes in cross sections of the skin after iontophoresis in vitro (a) and in vivo (b). The liposomes (Rho-lipo) consisted of an aqueous phase labeled with rhodamine dye (red) and a lipid membrane labeled with NBD (green). As a control, free rhodamine solution (Rho-soln) was also examined in vitro. Scale bar $=100 \mu \mathrm{m}$.

Figure 5. In vivo transfollicular delivery of liposomes encapsulating insulin via iontophoresis and fluorescent staining of micro vessels in rat skin

(a) The liposomes (INS-lipo) consisted of insulin labeled with rhodamine (red) and a lipid membrane labeled with NBD (green). As a control, free insulin labeled with rhodamine (INS-soln) was also examined. In the case of INS-lipo, we observed liposomes in cross sections of skin prepared either immediately or $18 \mathrm{hrs}$ after iontophoresis. Scale bar $=100 \mu \mathrm{m}$. (b) To examine the distribution of micro vessels in the skin, the cross sections of rat skin were stained by FITC-labeled GSI-B4 and Hoechst33342. The stained cross sections were observed using $15 \mu \mathrm{m}$-thick stacks of images obtained at $1 \mu \mathrm{m}$ intervals. Scale bar $=50 \mu \mathrm{m}$.

Figure 6. Effect of transfollicular delivery of insulin encapsulated in liposomes on blood glucose levels and plasma insulin levels in STZ-induced diabetic rats.

(a) The percentage changes from the initial blood glucose levels are shown. Closed squares or open triangles represent the group administered insulin encapsulated in liposomes (INS-lipo) $(n=4)$ or empty liposomes $(n=3)$ via iontophoresis, respectively. Open circles represent the group administered an i.p. injection of insulin solution $(\mathrm{n}=3)$. Data are expressed as mean \pm SD. (b) A graph showing plasma insulin levels at baseline ( $0 \mathrm{hr})$ and immediately (1 hr) and 
$18 \mathrm{hr}$ after iontophoresis of insulin encapsulated in liposomes (closed bars) ( $\mathrm{n}=7$ ) or empty liposomes (open bars) $(\mathrm{n}=5)$. As a positive control experiment, a $180 \mathrm{mg} / \mathrm{kg}(5 \mathrm{IU} / \mathrm{kg})$ dose of insulin solution was injected intraperitoneally in the diabetic rats (hatched bars) $(n=3)$. Data are expressed as mean $\pm \mathrm{SD} . \quad * \mathrm{p}<0.05, * * \mathrm{p}<0.01$ 

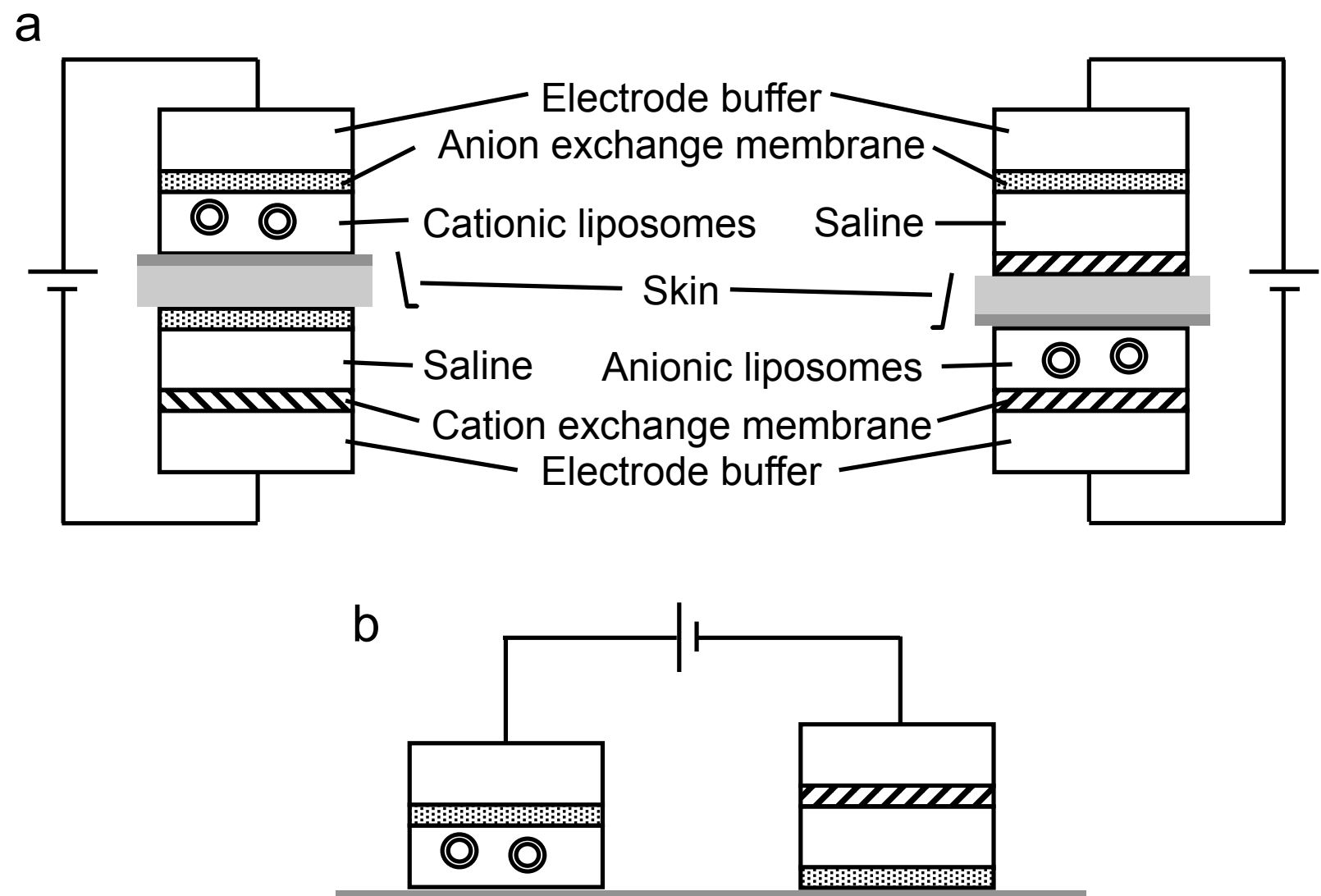

Fig. 1 
a

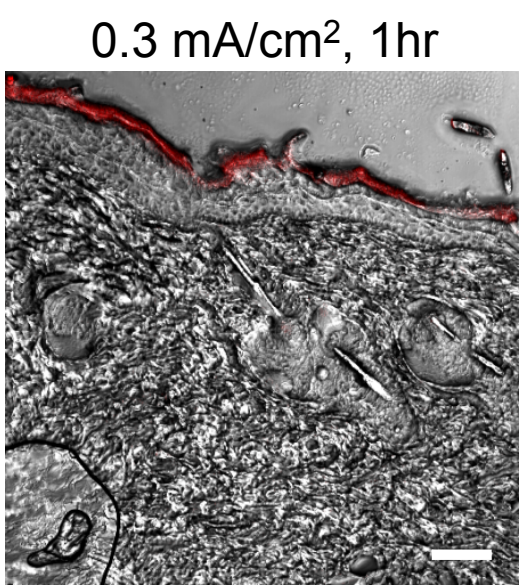

$0.3 \mathrm{~mA} / \mathrm{cm}^{2}, 2 \mathrm{hr}$
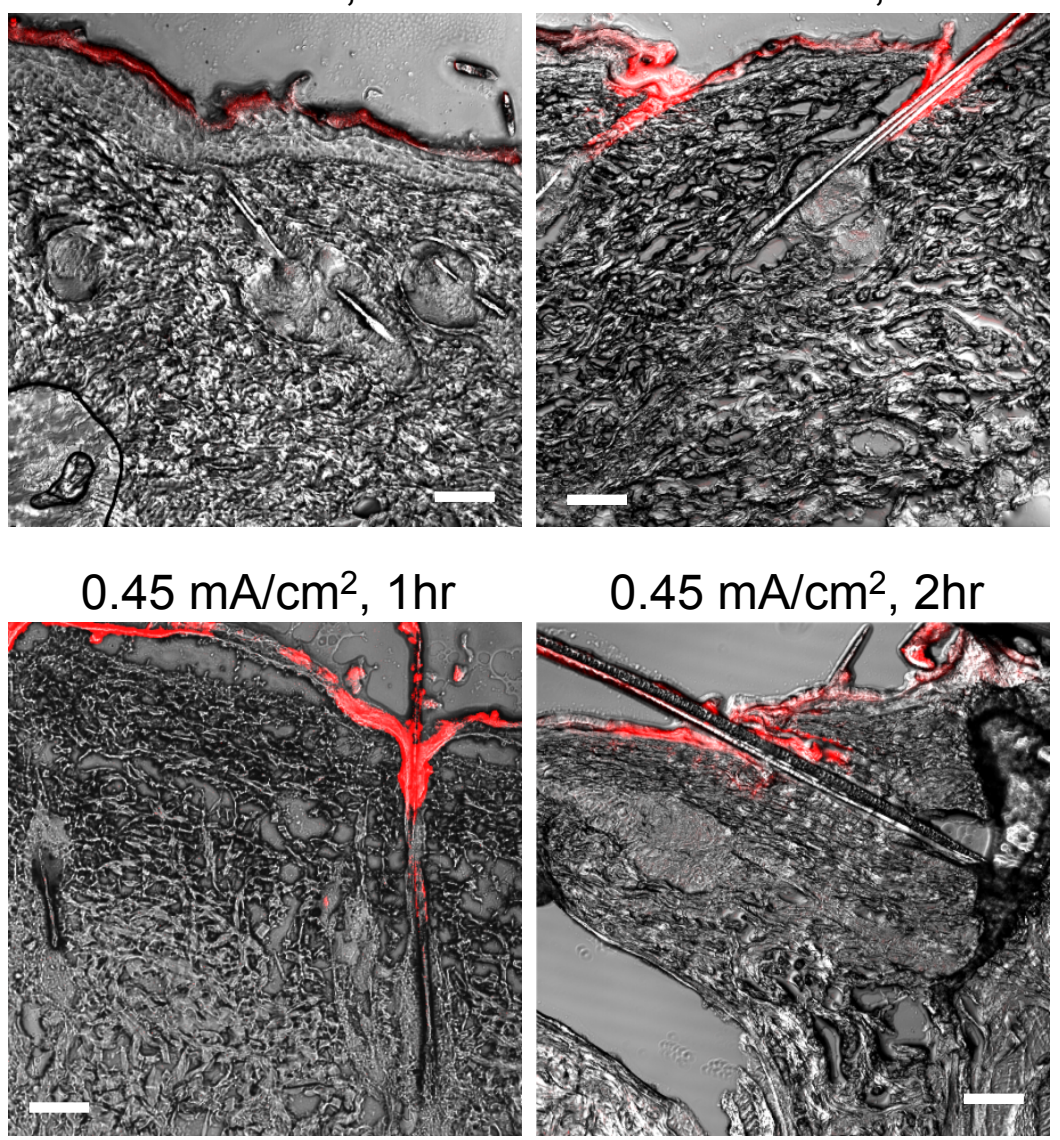

$0.45 \mathrm{~mA} / \mathrm{cm}^{2}, 2 \mathrm{hr}$

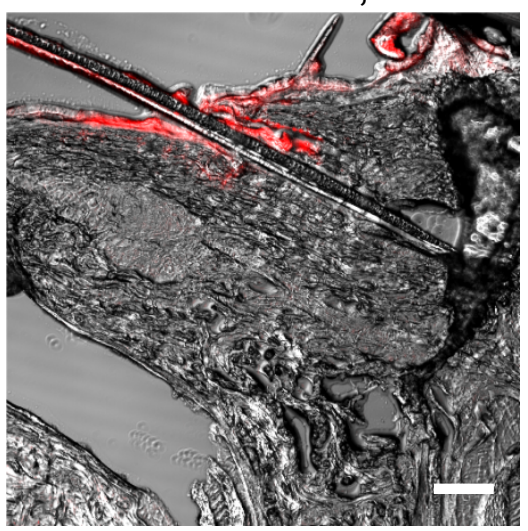

b

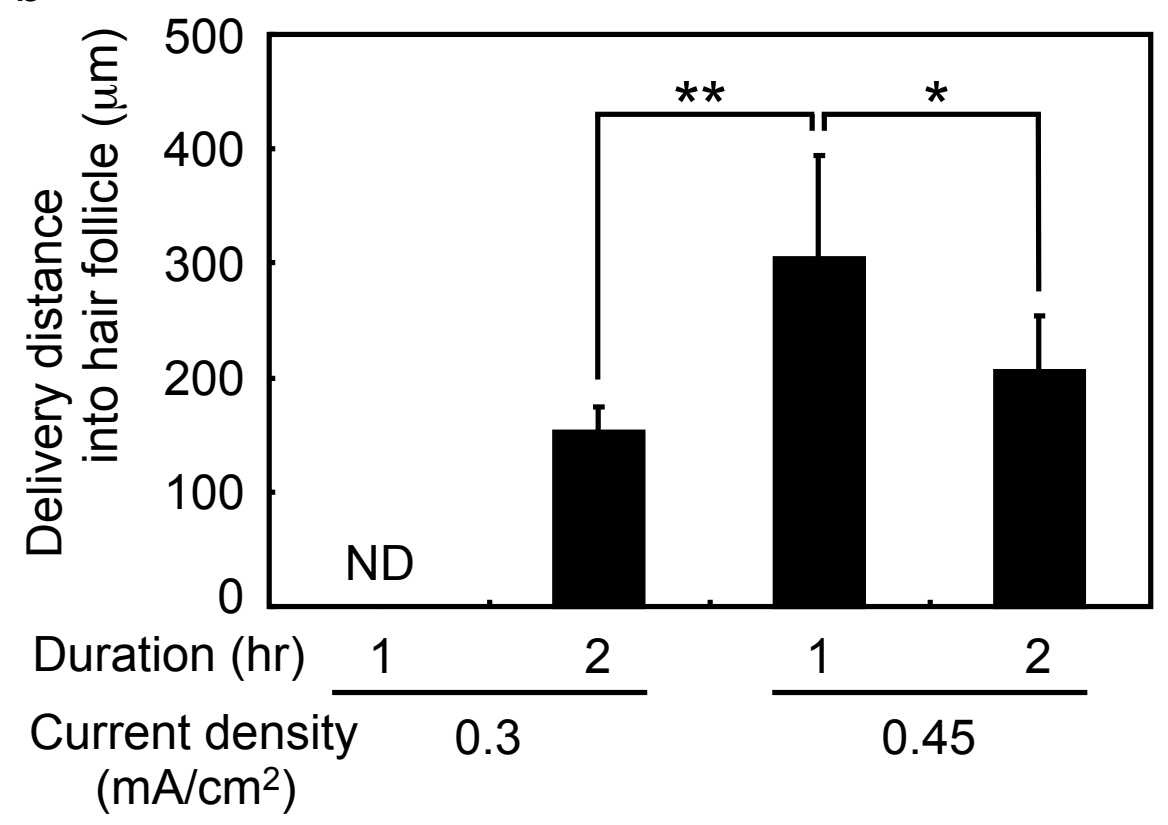

Fig. 2 
a

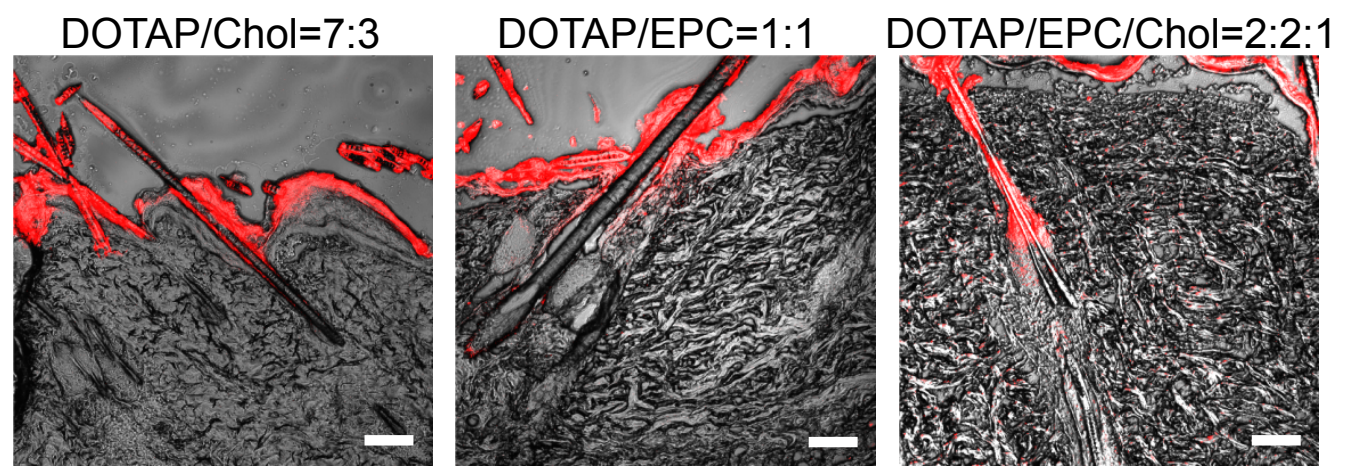

DOTAP/DOPE=1:1 DOTAP/EPC/DOPE=2:1:1

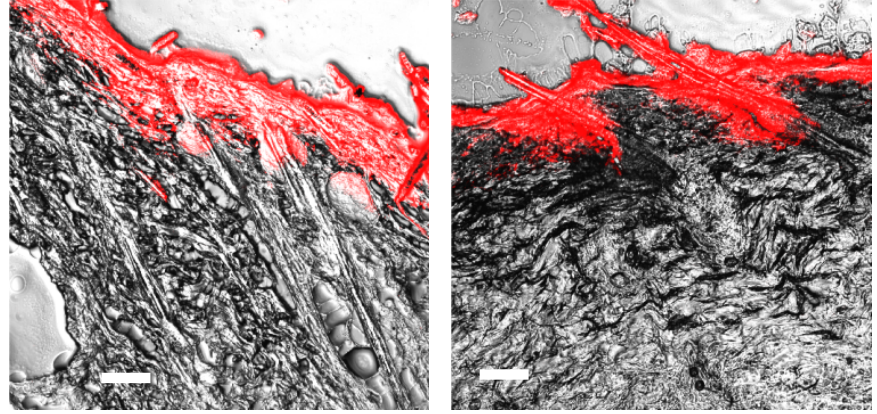

\section{CHEMS/EPC $=2: 9$}

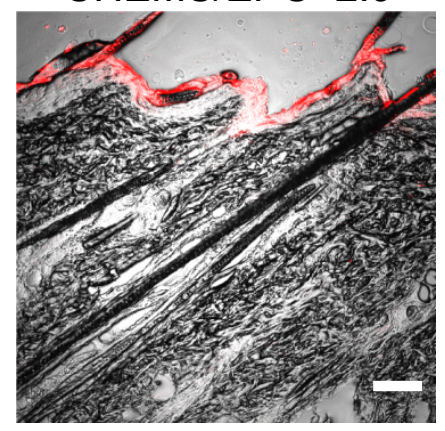

\section{CHEMS/DOPE $=2: 9$}

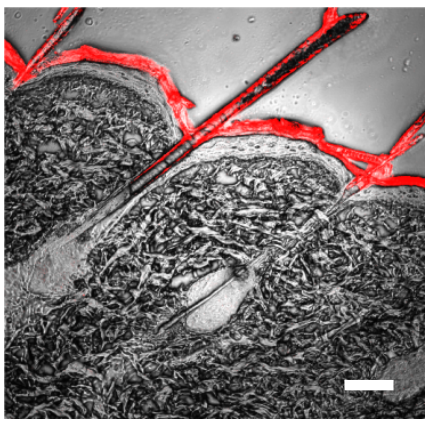

b

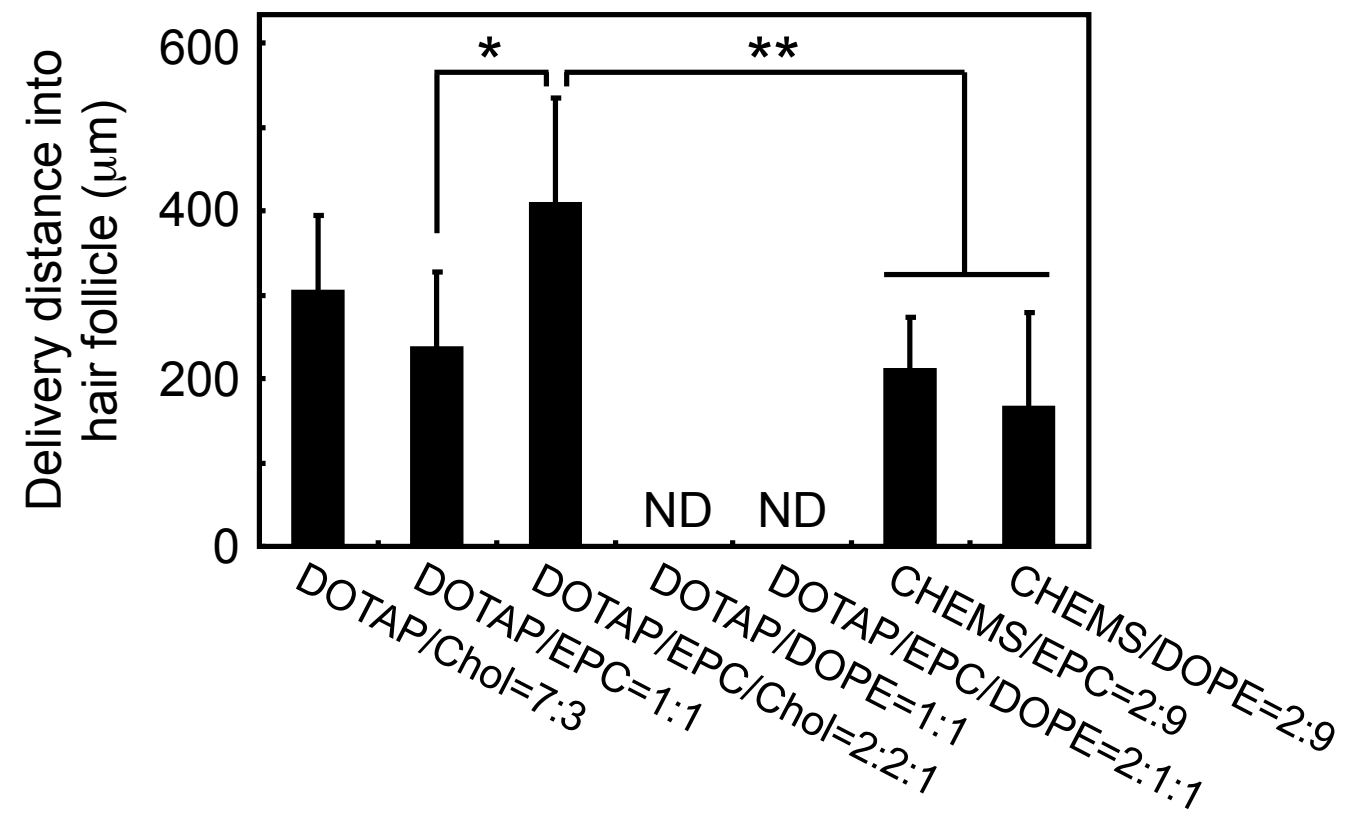

Fig. 3 
a
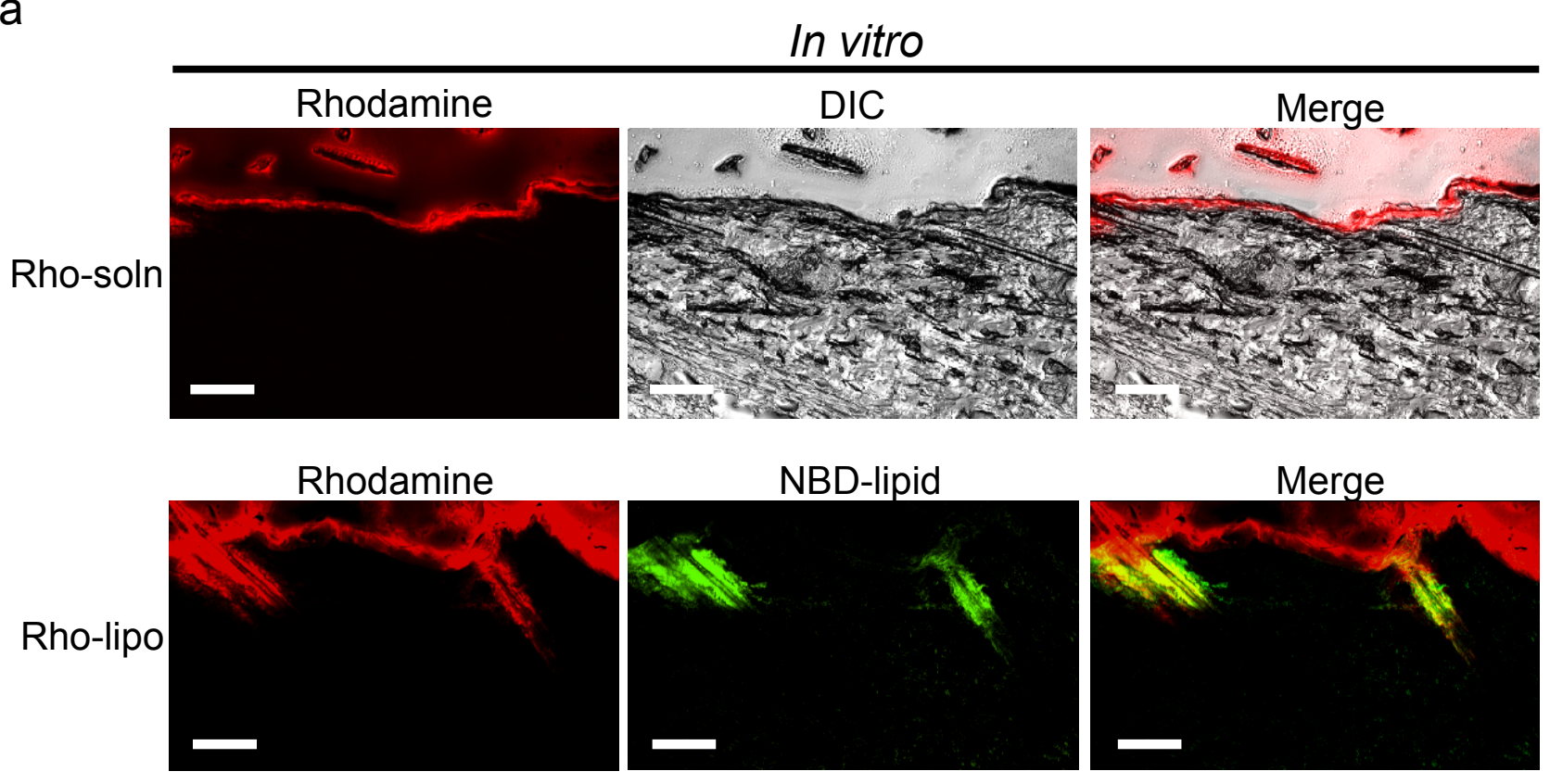

b

In vivo

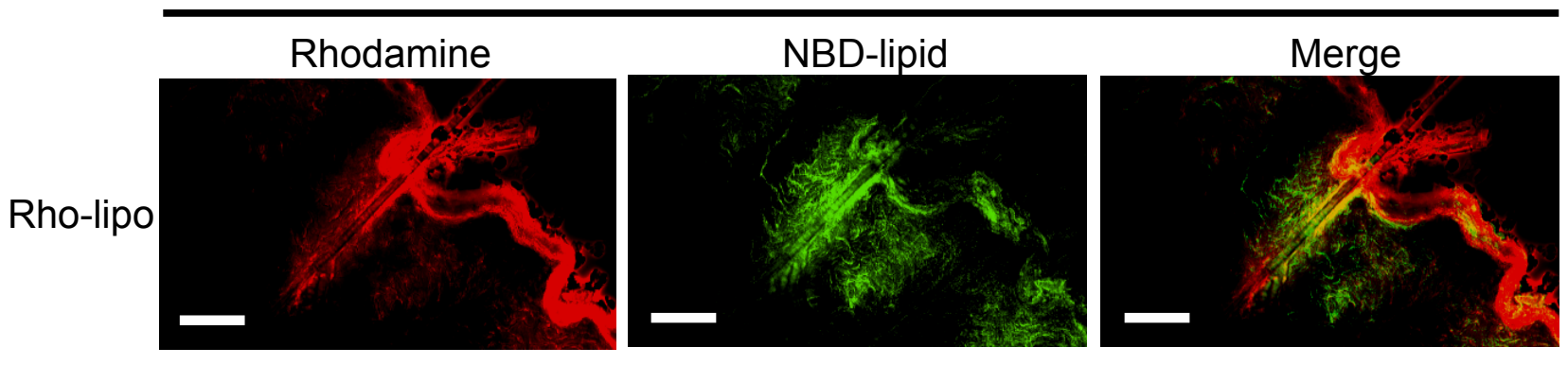

Fig. 4 
a
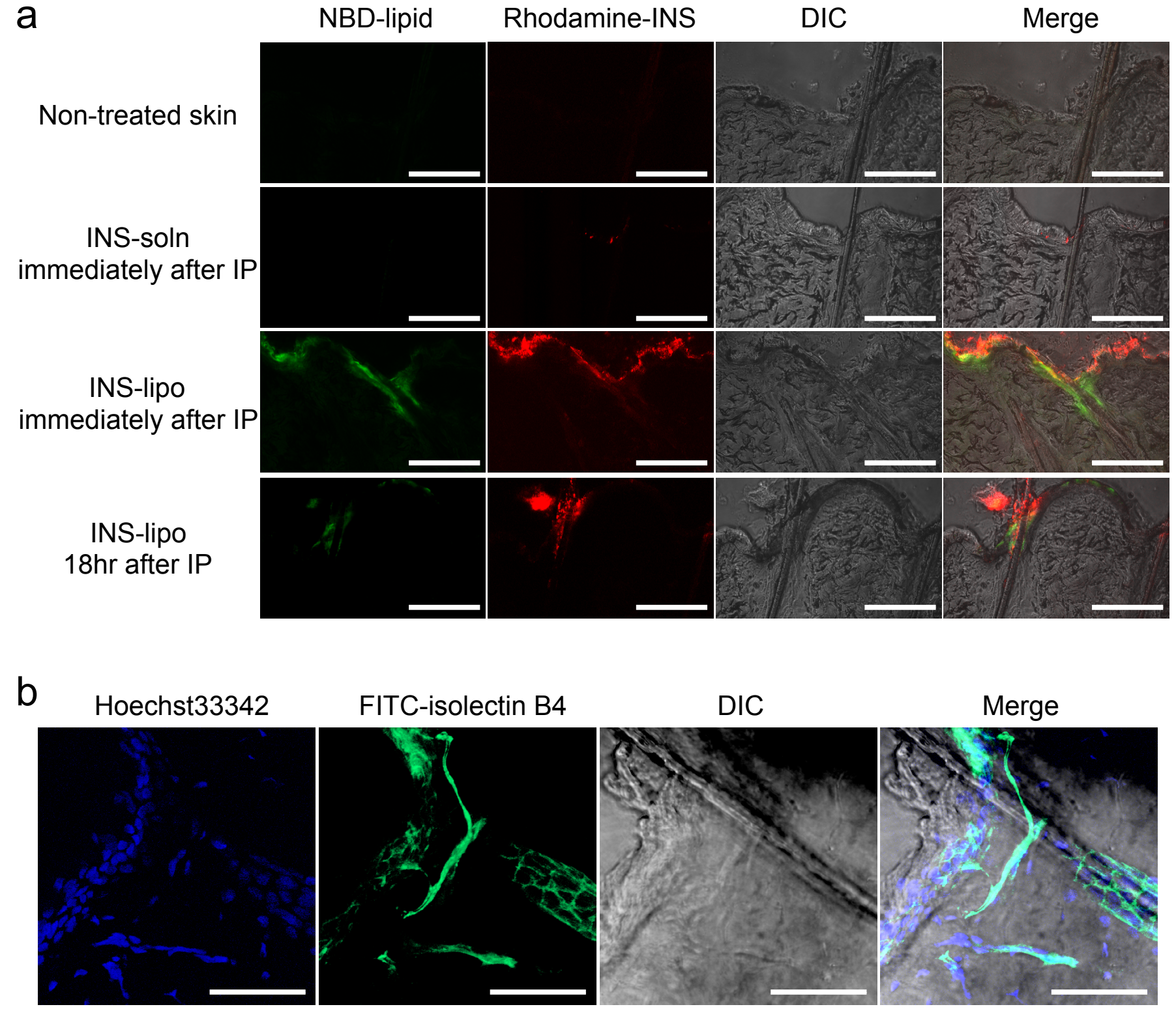

Fig. 5 

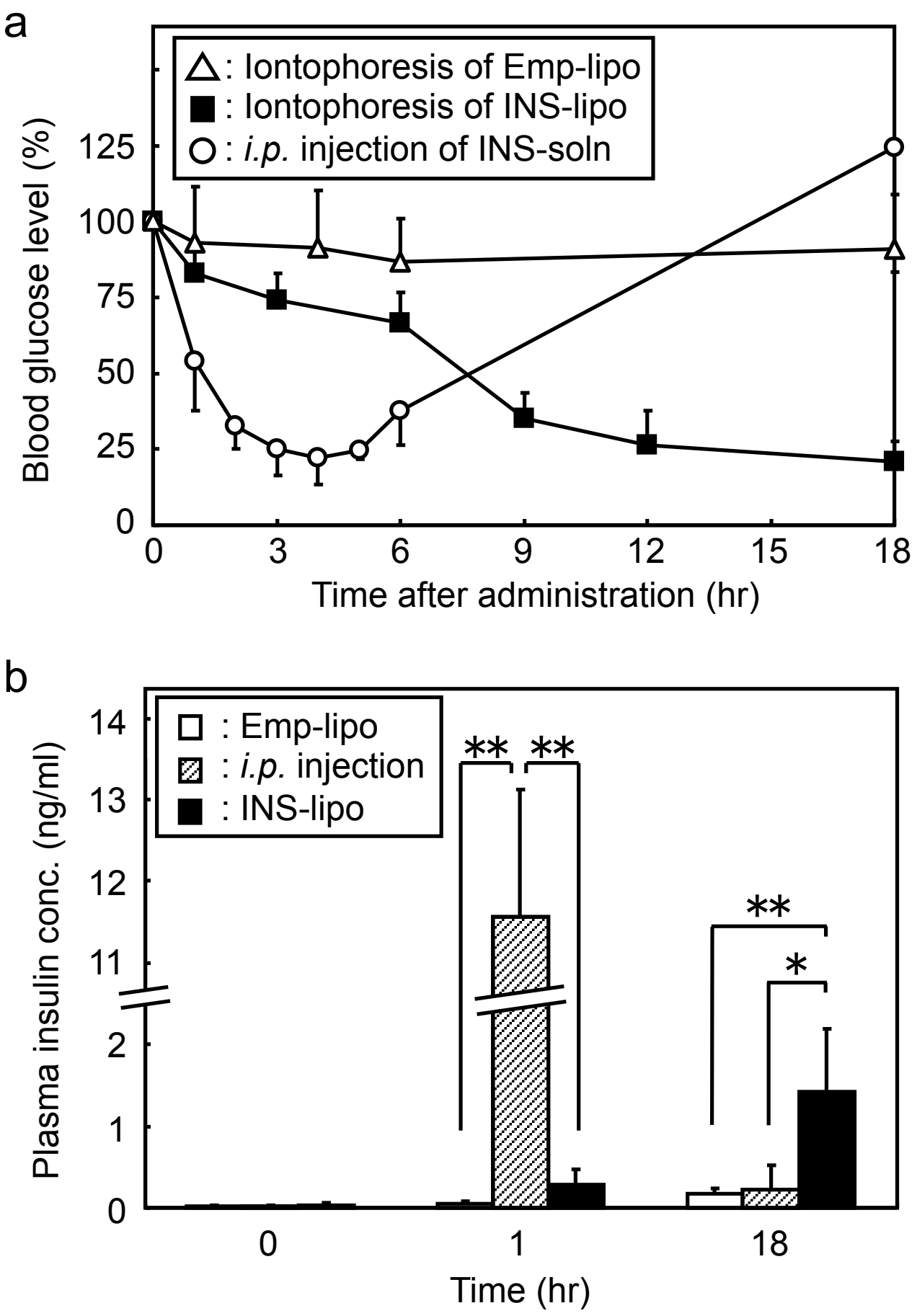

Fig. 6 